\title{
Linx
}

Revue des linguistes de l'université Paris X Nanterre

46 | 2002

Les connecteurs

\section{Par contre : un type particulier de dynamique discursive}

Badreddine Hamma et Pierre Patrick Haillet

\section{OpenEdition}

Journals

Édition électronique

URL : http://journals.openedition.org/linx/101

DOI : $10.4000 /$ linx.101

ISSN : 2118-9692

Éditeur

Presses universitaires de Paris Nanterre

Édition imprimée

Date de publication : 1 juin 2002

Pagination : 103-113

ISSN : 0246-8743

\section{Référence électronique}

Badreddine Hamma et Pierre Patrick Haillet, «Par contre : un type particulier de dynamique

discursive », Linx [En ligne], 46 | 2002, mis en ligne le 25 janvier 2011, consulté le 19 avril 2019. URL :

http://journals.openedition.org/linx/101 ; DOI : 10.4000/linx.101

Département de Sciences du langage, Université Paris Ouest 


\title{
Par contre : un type particulier de dynamique discursive
}

\author{
Badreddine Hamma, Université de Paris $X$ - Nanterre \\ \& Pierre Patrick Haillet, Université de Cergy-Pontoise
}

Né de la rencontre de deux études ${ }^{1}$ consacrées à par contre, cet article vise à apporter une contribution aux recherches sur le fonctionnement discursif de cette locution. Après une présentation succincte de son traitement traditionnel, on examinera un certain nombre d'exemples considérés comme représentatifs des différents emplois de par contre, et on en proposera une description unifiée dans le cadre de la «théorie de l'argumentation dans la langue »² développée par Ducrot et Anscombre. On tentera enfin d'expliquer la relation que par contre entretient, en français contemporain, avec en revanche.

\section{Par contre: approche normative et approche descriptive}

On sait que la locution par contre a longtemps été condamnée, et qu'elle l'a été en tant que combinaison de la préposition «par » avec la préposition « contre ». Voici, par exemple, la description qu'en donne Littré dans son édition de 1873 :

(1) Par contre est une locution dont plusieurs se servent, pour dire en compensation, en revanche : Si les artisans sont ordinairement pauvres, par contre ils se portent bien; Si le vin est cher cette année, par contre il est bon. Cette locution, qui a été tout particulièrement critiquée par Voltaire et qui paraît provenir du langage commercial, peut se justifier grammaticalement, puisque la langue française admet, en certains cas, de doubles prépositions [...], mais elle ne se justifie guère logiquement, par contre signifiant bien plutôt contrairement, en compensation et devant provenir de quelque ellipse commerciale (par contre ayant été dit pour par contre-envoi); en tout cas, il convient de suivre l'avis de Voltaire et de ne transporter cette locution hors du langage commercial dans aucun style.

\footnotetext{
${ }^{1}$ Il s'agit de la communication de P. P. Haillet, «Dictionnaires et "argumentation dans la langue" : PAR CONTRE, EN REVANCHE » au colloque international Dictionnaires de langue française et grammaire (Université de CergyPontoise, 21.03.2001), et des recherches conduites par B. Hamma sur « PAR » et « PAR CONTRE » à l'Université de Paris XNanterre (2000-2001).

2 Cf. Ducrot, 1972 ; Anscombre et Ducrot, 1976 ; Ducrot, 1980 ; Anscombre et Ducrot, 1977, 1983.
} 
A la même époque, Larousse (1876) en propose une description différente - en se plaçant du coup dans une tout autre perspective. S'y conjuguent deux considérations qui témoignent d'une attitude résolument « moderne », à savoir :

- l'attention accordée à l'usage répandu de par contre, à l'opposé d'une approche normative,

- l'analyse linguistique de par contre comme combinaison non pas de deux prépositions, mais de la préposition «par » avec un nom

C'est d'ailleurs dans la partie «substantif » - et non dans la partie «préposition»- de l'entrée « contre » que l'on trouve l'extrait reproduit ci-dessous :

(2) par contre, loc. adv. : en revanche, par compensation. Si plusieurs essais de Buffon sont beureux, quelques autres, par contre, ne le sont pas. // Cette locution, généralement condamnée par les grammairiens, est universellement usitée. Il n'est d'ailleurs pas impossible de la justifier, en admettant que contre y est pris substantivement, ce que prouve surabondamment la présence de la préposition par.

Ces deux traitements radicalement opposés nous semblent représentatifs des deux attitudes à l'égard de par contre; si dans des ouvrages plus récents la prise en compte de l'usage prévaut globalement - sur sa condamnation, il subsiste encore des traces du traitement que lui ont si longtemps réservé les puristes. C'est ce que permet de constater, par exemple, l'examen de l'entrée correspondante du dictionnaire de l'Académie (édition de 1988); bannie des éditions précédentes, la locution y est accompagnée du commentaire reproduit ci-dessous :

(3) par contre: en revanche, d'un autre côté, en contrepartie, en compensation, à l'inverse. Condamnée par Littré, d'après une remarque de Voltaire, la locution adverbiale par contre a été utilisée par d'excellents auteurs français, de Stendhal à Montherlant, en passant par Anatole France, Henri de Régnier, André Gide, Marcel Proust, [...] Paul Morand, Antoine de Saint-Exupéry, etc. Elle ne peut donc être considérée comme fautive, mais l'usage s'est établi de la déconseiller chaque fois que l'emploi d'un autre adverbe est possible.

Les deux traitements présentent par ailleurs un point commun : sur le plan des synonymes indiqués, en compensation, par compensation et en revanche sont systématiquement suggérés tant dans les ouvrages déjà cités - cf. exemples (1) à (3) - que dans le Dictionnaire du Français Contemporain (1980), Le Robert Méthodique (1989), Le Petit Robert (1996), etc. Avant de revenir, dans la section 3 de cette étude, sur la relation entre par contre et en revanche, arrêtons-nous un instant sur l'idée même de «compensation »; si elle est à coup sûr présente dans «par contreenvoi » signalé par Littré, ou encore dans :

(4) Le magasin est assez exigu, par contre il est bien situé. (Le Robert Méthodique, 1989)

elle ne l'est certainement pas, par exemple, dans :

(5) C'est un garçon charmant; par contre, son frère a un caractère détestable. (Dictionnaire $d u$ Français Contemporain, 1980)

C'est, précisément, la fréquence de ces deux types d'emplois de par contre - illustrés par (4) et (5) cidessus - qui nous conduit à en proposer une approche différente, visant à circonscrire l'invariant commun à l'ensemble de ses occurrences. Notre démarche s'inscrit dans le cadre de la « théorie de l'argumentation dans la langue ». 
Par contre : un type particulier de dynamique discursive

\section{2. «Théorie de l'argumentation dans la langue »}

\section{1. Mais, or et « dynamique discursive ॥}

On le voit, si l'élément qu'introduit par contre apparaît comme «compensant » ce qui précède dans certains exemples, il en est d'autres qui n'admettent pas cette interprétation - d'où l'impossibilité de fonder la description de par contre sur l'idée de «compensation ». Sur le plan du principe, il s'agit d'un phénomène comparable à celui que met en évidence Ducrot (1972, p. 129) à propos de la conjonction mais: en effet, à côté de «Il est républicain, mais honnête ", on a «Il faisait beau, mais jétais fatigué », et s'il est possible d'avancer que le locuteur du premier exemple exprime «lincompatibilité » entre "être républicain » et "être honnête », il n'en va certainement pas de même pour la relation entre "il faisait beau » et "jétais fatigué ». Ce qui conduit à caractériser mais "non pas par le contenu de ce qui l'entoure [...], mais par la nature des rapports ou des enchânements qu'il introduit » (Ducrot et al., 1976/1980, p. 96). L'exemple de la conjonction mais nous servira ici à illustrer l'application des principes de la «théorie de l'argumentation dans la langue ».

Mais fait partie des connecteurs, "mots dont le rôle habituel est d'établir un lien entre deux entités sémantiques. [...] [L]es connecteurs de la langue concernent non point des segments matériels du texte, mais des entités sémantiques qui peuvent n'avoir qu'un rapport très indirect avec de tels segments. » (Ducrot, 1980, p. 15). Pour illustrer la distinction ainsi opérée entre « segments matériels du texte » et «entités sémantiques », considérons l'exemple suivant :

(6) Ce vin est cher, mais il est bon.

Dans le cadre de la « théorie de l'argumentation dans la langue », les segments de discours reliés par mais sont considérés comme deux arguments favorisant chacun un type de conclusions (explicites ou implicites). Ces deux classes de conclusions constituent les entités sémantiques que le locuteur de «A, mais B » représente comme contraires. Ainsi, le fonctionnement discursif de mais dans (6) sera schématisé comme suit : «A »- soit «Ce vin est cher »- est représenté comme un argument pour une conclusion du type « $\mathrm{C} »-$ par exemple: «Je n'en bois pas souvent», «Je ne vais pas en racheter », etc., et « B »- soit « il est bon»-constitue alors un argument en faveur de la conclusion opposée « non $\mathrm{C}$ » du type ${ }^{3}$ : « J'en bois souvent », « Je vais en racheter », etc.

En outre, l'examen des enchaînements discursifs admis et exclus montre que «A mais B » est globalement orienté vers les conclusions favorisées par «B », c'est-à-dire le second argument :

(7) Ce vin est cher, mais il est bon. Je vais donc en racheter.

Ce vin est cher, mais il est bon. *Je ne vais donc pas en racheter.

(8) Ce vin est bon, mais il est cher. Je ne vais donc pas en racheter. Ce vin est bon, mais il est cher. *Je vais donc en racheter.

\footnotetext{
${ }^{3}$ Seule compte la relation d'opposition entre les deux types de conclusions favorisées respectivement par «A » et par « $\mathrm{B}$ »; on pourra donc tout aussi bien avoir, par exemple, « il ne se vend pas bien » comme représentatif du type « $\mathrm{C}$ » et « il se vend bien » comme représentatif du type « non $\mathrm{C} »$, etc.
} 
En d'autres termes, l'emploi de mais a pour conséquence l'incongruité de certains enchainements, en même temps qu'il en privilégie d'autres ${ }^{4}$ - ce qui revient à dire qu'il oriente le discours ; nous dirons qu'il reflète la dynamique discursive adoptée par le locuteur.

Prenons maintenant un autre exemple d'analyse fondée sur ce principe. On constate, à propos de la conjonction or, qu'elle peut relier tant des segments entre lesquels on perçoit intuitivement une relation d'opposition, comme dans :

(9) Tu m'as dit que tu serais à la bibliothèque, or tu n'y étais pas. (Dictionnaire HachetteOxford, 1994)

(10) Vous croyez avoir raison, or vous n'avez rien prouvé. (Le Petit Robert, 1996)

que des segments entre lesquels une telle relation semble impossible à envisager :

(11) Tous les hommes sont mortels, or je suis un homme, donc je suis mortel. (Dictionnaire Hachette-Oxford, 1994)

(12) Commentaire d'un ministre de la gauche plurielle à propos des affaires de la Mairie de Paris: "On n'a pas intérêt à ce que Chirac soit mis en cause. Si cétait le cas, nous serions dans une situation à l'américaine. Or, pour réussir notre politique, nous avons besoin de stabilité."

(13) Les musées sont fermés le mardi, or c'était justement un mardi. (Dictionnaire HachetteOxford, 1994)

phénomène qui a pour corollaire la possibilité de remplacer or par mais dans (9) et (10), mais non dans (11), (12) et (13).

Parallèlement à l'impossibilité de faire intervenir la notion d'opposition dans la description de l'invariant de or, on constate - sur le plan de la dynamique discursive - que dans les deux types d'emplois, ce connecteur met un élément en rapport avec ce qui précède à des fins argumentatives, que ce soit pour appuyer une conclusion déjà énoncée ou pour en annoncer une (explicite ou implicite). Là où il commute avec mais, son emploi détermine l'orientation argumentative globale du discours vers la conclusion favorisée par l'élément qu'il introduit ; là où son remplacement par mais n'est pas possible, il introduit un complément indispensable du raisonnement, la conclusion visée étant appuyée par la mise en relation du segment qui suit or avec celui qui le précède. Que ce second segment soit ou non perçu par ailleurs comme opposé au premier, l'emploi de or a invariablement pour effet de représenter l'argument introduit comme décisif ${ }^{5}$ pour la dynamique discursive adoptée par le locuteur.

C'est dans cette perspective que nous nous proposons d'examiner le fonctionnement discursif de par contre; il s'agira de déterminer - indépendamment de la nature des segments de discours que relie ce connecteur - quel type de dynamique met invariablement en place son emploi.

\footnotetext{
${ }^{4}$ De la même manière, on a «Je te recommande ce vin : il est cher, mais bon », mais non « Je te recommande ce vin : il est bon, mais cher ».

5 Cf. Haillet, 2000.
} 
Par contre : un type particulier de dynamique discursive

\section{2. «A, par contre $B »$}

Nous adoptons ici la notation schématique «A, par contre B», étant bien entendu qu'en discours, la configuration étudiée peut se manifester matériellement de diverses façons :

(14) Le vin blanc est bon; par contre, le vin rouge pique un peu.

(15) J'ai acheté le bouquin ; il n'y a rien sur l'exclamation, par contre.

(16) Il n'a pas eu son permis ; il a, par contre, rencontré l'amour de sa vie.

(17) Par contre, la Volvic, il va falloir la sortir !

Nous considérons que l'emploi de par contre a pour effet de mettre en relation un segment de discours « $\mathrm{B}$ » (respectivement, «le vin rouge pique un peu», «il n'y a rien sur l'exclamation », "il a rencontré l'amour de sa vie ») avec un segment de discours «A » ( Le vin blanc est bon», " Jai acheté le bouquin», "Il n'a pas eu son permis»). L'exemple $(17)^{6}$ illustre le cas où le segment ${ }^{7}$ «B»- «la Volvic, il va falloir la sortir!»- est mis en relation avec une entité sémantique qui ne correspond pas à un segment « $\mathrm{A}$ » explicite ; cet énoncé, produit par une caissière de supermarché qui s'adresse à un client ayant mis sur le tapis roulant tous ses achats à l'exception d'un pack de six bouteilles de Volvic, resté dans le caddie, s'oppose en fait à une entité sémantique paraphrasable par « il n'est pas nécessaire de sortir du caddie les packs de Cristaline », autre marque d'eau minérale qui jouit dans cette chaîne de magasins d'un statut particulier.

Pour tenter de circonscrire la nature exacte de la relation qu'instaure entre « $\mathrm{A}$ » et « $\mathrm{B}$ » l'emploi de par contre, nous avons écarté les exemples - assez fréquents - où il se combine avec mais, par souci de ne pas confondre les effets de sens produits respectivement par l'un et par l'autre ; pour les mêmes raisons, nous n'avons pas non plus retenu les combinaisons de par contre avec $s i^{8}$ dit «adversatif », «concessif » ou encore « contrastif »- comme celles illustrées par (1), extrait de Littré (1873) et par (2), tiré de Larousse (1876).

L'examen d'un corpus d'énoncés attestés - écrits et oraux - nous conduit à avancer que l'emploi de «A, par contre B » a invariablement pour effet d'opposer, avec plus ou moins de force, « $B$ » à « $A$ », de telle sorte que les conclusions favorisées par « $A$ »se trouvent, à un extrême, équilibrées par celles que privilégie « $\mathrm{B}$ », et à l'autre, restreintes du fait de « $\mathrm{B}$ ». Ainsi, le premier cas est illustré par (4):

\section{(4) Le magasin est assez exigu, par contre il est bien situé.}

Ce que le locuteur de (4) dit sur l'endroit où se situe le magasin est de nature à favoriser un type déterminé de conclusions, telles que : « le prix est justifié », « je vous en recommande l'acquisition », etc., et rétablit ainsi l'équilibre par rapport à celles que l'on pourrait tirer de l'assertion relative à l'exiguité des lieux. Rien de tel dans (18), extrait d'un article consacré à la création d'emplois observée dans le Val d'Oise en 1999:

(18) En 1999, les emplois salariés privés ont progressé de 6,4\% (+ 14578 emplois) : la meilleure performance des départements français, devant les Yvelines, la Vendée et l'Ille-et-Vilaine. [... ]

\footnotetext{
6 Ainsi que ses variantes « La Volvic, par contre, il va falloir la sortir ! » et « La Volvic, il va falloir la sortir, par contre ! ».

${ }^{7}$ Notons que ce segment n'est pas toujours introduit par par contre au sens purement syntaxique.

${ }^{8}$ Sur si, cf. Anscombre, 1985, p. 347-348.
} 
Cergy-Pontoise et l'Est valdoisien sont en tête. Plus de la moitié des nouveaux emplois a été créée à Cergy-Pontoise (7 421), et plus du tiers dans l'Est du département, (4 955). La Plaine de France (519) et les Rives de Seine (1 227) ont aussi obtenu de bons résultats. Par contre, la Vallée de Montmorency, la Vallée de l'Oise et le Vexin n'ont pas enregistré de variation significative du nombre d'emplois salariés en 1999.

« Par contre $B$ » a ici pour effet de marquer une restriction quant à la portée de certaines conclusions que l'on pourrait tirer de «A », du type : «des emplois ont été créés partout dans le Val d'Oise »sans que le discours soit pour autant réorienté globalement vers les conclusions favorisées par « B». C'est un fonctionnement similaire qui se manifeste dans :

(19) Je m'en suis sortie, je vis seule depuis mon divorce. Une « relation suivie »? non, je ne veux pas revivre un deuxième échec ; ça, par contre, je ne pourrais pas supporter.

où «par contre $\mathrm{B}$ » restreint la portée des conclusions du type « je suis forte », favorisées par "je m'en suis sortie, je vis seule depuis mon divorce »; l'effet de sens produit est paraphrasable par « je ne suis pas si forte que cela, pas au point de... ». De même, dans :

(20) Un avocat vous sera commis d'office. Par contre, vous ne pourrez vous entretenir avec lui qu'à partir de la vingtième heure.

une nuance est apportée à la bonne nouvelle communiquée à la personne placée en garde à vue ; là encore, l'orientation argumentative globale n'est pas inversée pour autant.

Nous avons vu, dans la section 1, que l'emploi de «par contre B» n'induit pas nécessairement une idée de « compensation » de « A » par « B »; les exemples (18) à (20) confirment cette constatation. Pour caractériser la relation qui s'établit invariablement entre « B » et « $A$ » dans «A, par contre $B$ », il est, nous semble-t-il, préférable de parler de « contrepartie » 9 - au sens où ce terme est défini dans Le Petit Robert (1996) : «chose qui s'oppose à une autre en la complétant ou en l'équilibrant».

Ce terme nous semble particulièrement adéquat pour décrire le rôle que le segment «B» joue par rapport au segment « $\mathrm{A} »$ dans la dynamique discursive mise en place du fait de l'emploi de «par contre $B$ ». Considérer que « $B »$ constitue une contrepartie - discursive - de « $A$ » permet de rendre compte tant des cas où « par contre $B$ » ne fait que restreindre la portée de « $A$ », que de ceux où «par contre B» établit l'équilibre entre les deux termes de la relation ainsi instaurée, en les mettant à égalité. Dans ce dernier type de configurations, on a souvent une conclusion explicite qui s'appuie exclusivement sur l'existence d'une telle contrepartie, sans que «A » ou «B » apparaissent comme «privilégiés » :

(21) Cet inventaire des diverses possibilités explique que l'on trouve tant de différences entre les peuples: ainsi, en Polynésie, il n'existe pas en général de rites de puberté, l'enfant devient progressivement adolescent, ce n'est que par le mariage qu'il passe à l'état adulte et ce sont ici les cérémonies du mariage qui sont prépondérantes ; par contre, en Mélanésie, c'est le passage de l'enfance à l'adolescence qui est abrupt, et les rites de mariage n'ont plus la même importance. (ex. emprunté à Wiederspiel et Masseron, 2001)

\footnotetext{
${ }^{9}$ Ce qui, précisons-le, ne signifie nullement que nous considérons le syntagme « en contrepartie » comme susceptible d'être substitué à par contre dans tous ses emplois. Notre réflexion ne porte que sur la nature de la relation discursive qu'institue entre « B » et « $A$ » n'importe quelle occurrence de « $A$, par contre $B$ ».
} 
Ce qui étaye, ici, l'assertion relative aux «différences entre les peuples », c'est précisément la mise en relation de l'exemple de la Polynésie avec sa contrepartie, celui de la Mélanésie.

La représentation de « $B$ » comme une contrepartie discursive de « $A$ » constitue, dans notre approche, l'invariant commun à l'ensemble des emplois de « A, par contre B ». A cet invariant, défini comme un type particulier de dynamique discursive adoptée par le locuteur, peuvent s'ajouter d'éventuels effets de sens secondaires, en fonction de la nature des segments « $A$ » et « B ».

\section{Par contre et en revanche}

Nous nous proposons d'examiner, dans cette section, le fonctionnement discursif de en revanche et de le comparer à celui de par contre. Nous adoptons, là encore, la notation schématique «A, en revanche B » qui inclut par convention les différents cas qui se présentent en discours sur le plan de la distribution :

(22) La moisson a été médiocre, en revanche la récolte des fruits a été excellente. (Dictionnaire $d u$ Français Contemporain, 1980)

(23) C'est le plus jeune joueur de l'équipe... et très doué, en revanche !

(24) Le manque de «lisibilité » des tarifs, dont la diversité, conséquence de la concurrence, entretient une opacité parfois calculée, est un vrai «sujet de préoccupation». La bataille de la transparence ne fait que commencer.

La surfacturation des appels entrants, les plus juteux puisqu'ils représentent le tiers des recettes des opérateurs et déterminent la rentabilité de leur investissement, est en revanche en voie d'être résorbée.

L'observation d'un corpus nous conduit à constater des similitudes entre par contre et en revanche non seulement pour ce qui est de leur distribution, mais également en ce qui concerne le type de dynamique discursive mise en place du fait de leur emploi. Ainsi, dans (22), les résultats de la récolte des fruits sont représentés comme une contrepartie de la médiocrité de la moisson ; le locuteur de (23) exprime une relation discursive du même type entre les talents du joueur et son jeune âge; enfin, l'exemple (24) - extrait d'un article sur la téléphonie mobile - illustre une dynamique discursive comparable, l'assertion relative à la baisse du coût des appels entrants étant mise en rapport avec ce qui est dit sur l'opacité des tarifs.

Doit-on en conclure que en revanche fonctionne comme une « variante soutenue » de par contre, susceptible de lui être substituée dans n'importe quel emploi?

Pour tenter de répondre à cette question, rappelons tout d'abord quelques critiques formulées à l'égard de l'approche de type normatif - évoquée dans la section 1 - qui consiste à préconiser l'usage systématique de en revanche au lieu de par contre. Grevisse (1964, p. 994) dit notamment :

Il ne faudrait pas croire que en compensation ou en revanche puissent, dans tous les cas, suffire pour exprimer l'idée qu'on rendrait au moyen de par contre: en compensation et en revanche ajoutent à l'idée d'opposition une idée particulière d'équilibre beureusement rétabli ; par contre exprime, d'une façon toute générale, la simple opposition et a le sens nu de «mais d'autre part », 
Badreddine Hamma \& Pierre Patrick Haillet

" mais d'un autre côté ». — Gide le fait très justement remarquer : "Trouveriez-vous décent qu'une femme vous dise: "Oui, mon frère et mon mari sont revenus saufs de la guerre; en revanche j'y ai perdu mes deux fils»? ou: «La moisson n'a pas été mauvaise, mais en compensation toutes les pommes de terre ont pourri » ?

Une position similaire est exprimée dans Le Robert analogique et alphabétique (1981):

PAR CONTRE est condamné par certains puristes; cependant il n'est pas toujours remplaçable. Il introduit un avantage ou un inconvénient. EN COMPENSATION et EN REVANCHE n'introduisent qu'un avantage. Si on peut les employer dans la phrase «Sil n'a pas de cœur, par contre il est intelligent », il est impossible de les substituer à PAR CONTRE dans celle-ci : «Sil est intelligent, par contre il n'a pas de cœur».

Les notions d'avantage et d'inconvénient - tout comme l'idée d'équilibre « heureusement rétabli »étant de nature subjective, l'intégration de ce principe dans une description de en revanche issue de la «théorie de l'argumentation dans la langue » consisterait à dire que l'emploi de « $\mathrm{A}$, en revanche $\mathrm{B} »$ revient, entre autres, à représenter $« \mathrm{~B} »$ comme un avantage pour le locuteur ${ }^{10}$; c'est cette contrainte qui expliquerait alors l'impossibilité de substituer en revanche à par contre dans des énoncés du type « Mon frère et mon mari sont revenus saufs de la guerre ; par contre, j'y ai perdu mes deux fils ».

Or, si (22), (23) et (24) illustrent bien ce principe, on constate cependant que de nombreux exemples mettent en difficulté une telle conception de en revanche. Ainsi, l'emploi de « en revanche $\mathrm{B} »$ n'a pas pour effet de représenter «B $\mathrm{B}$ comme quelque chose de positif pour le locuteur dans :

(25) Les enquêtes d'opinion effectuées ces dernières années sur le thème de la construction européenne font régulièrement apparaitre un partage « deux tiers / un tiers » entre partisans et adversaires de l'Europe. On observe, dans cette enquête, une permanence de ce rapport de force. Ainsi, 68 \% des Français considèrent que l'Union Européenne est plutôt une bonne chose pour leur pays, contre $23 \%$ qui expriment un avis contraire. En revanche, ils sont un peu moins nombreux à penser que l'Europe est une bonne chose pour les gens comme eux (64\% contre $23 \%$ ).

(26) Au-delà de cette volonté de progression générale du rythme de la construction européenne, les Français souhaitent la mise en place de structures communes à l'ensemble des Européens dans de nombreux domaines à l'exception du domaine politique. Ils plébiscitent avant tout la mise en œuvre d'une Europe de «la sécurité » (défense, justice, police) et de l'Europe monétaire.

$74 \%$ souhaitent l'existence d'un seul système de défense européen. Même les «antieuropéens » s'y montrent favorables. Il convient de noter qu'en revanche seulement $58 \%$ des interviewés se prononcent pour une politique étrangère commune.

« $\mathrm{B}$ » est représenté comme une contrepartie ${ }^{11}$ discursive de « $\mathrm{A} »$; on a ici le même type de dynamique que dans (18) ou (19) avec par contre. C'est ce que l'on voit également dans :

\footnotetext{
10 Ce qui vaudrait également pour « A, en compensation B ». Il s'agit de ce que l'on constate à propos de « grâce à », qui introduit la cause d'une conséquence représentée de ce fait comme «positive pour le locuteur» - formule qui permet de rendre compte, par exemple, de « Grâce à la grève du métro, la réunion a été annulée ».

${ }^{11}$ A noter que le Dictionnaire du Français Contemporain (1980), ainsi que Le Robert Méthodique (1989), proposent en contrepartie comme expression synonymique de en revanche.
} 
(27) On vient d'apprendre que le procureur général a requis 5 ans de prison et 2 MF d'amende contre Sirven et Le Floch-Prigent. On ne connaît pas encore, en revanche, le réquisitoire à l'encontre de Roland Dumas et de Christine Deviers-Joncour.

Ce commentaire donné à la fin du Journal télévisé de France 2 illustre, nous semble-t-il, lui aussi un fonctionnement discursif similaire à celui de par contre : sans que les segments « $A$ » et « $B$ » soient ici représentés comme positifs - ou négatifs - pour le locuteur, l'emploi de « en revanche B » a pour effet de restreindre la portée des conclusions que l'on pourrait tirer de l'annonce des peines requises contre Sirven et Le Floch Prigent. Enfin, le même type de phénomène se manifeste dans :

(28) Aujourd'hui, l'Afghanistan ne cultive plus le pavot; la pression internationale s'est révélée efficace. En revanche, elle n'a pu empêcher la destruction des statues de Bouddha par les talibans.

où il serait pour le moins incongru de considérer que « B » représente un avantage pour le locuteur en l'occurrence, un journaliste français qui termine ainsi son reportage sur l'Afghanistan. Là encore, l'emploi de « en revanche $\mathrm{B}$ » reflète une dynamique discursive consistant à représenter « B » comme une contrepartie de «A».

Pour tenter d'expliquer cette similitude de fonctionnement discursif que l'on observe entre en revanche et par contre, nous voudrions avancer une hypothèse d'ordre sociolinguistique. Partons du constat qu'il y a variation : les sujets parlants adoptent, grosso modo, deux types d'attitudes qui nous semblent correspondre aux deux approches de par contre évoquées dans la section 1. A côté de locuteurs pour qui rien ne s'oppose à l'usage de cette locution, et qui n'éprouveraient donc aucune gêne à l'employer, par exemple, dans (22) à (28), on a ceux qui - marqués plus ou moins consciemment par sa condamnation - lui préfèrent en revanche qu'ils considèrent comme sa "variante soutenue ». L'hypothèse que nous faisons est que ce second groupe a globalement tendance à utiliser en revanche plutôt que par contre non seulement à l'écrit, mais également à l'oral - surtout lorsqu'ils se trouvent sur un plateau de télévision... Un dernier exemple illustrera notre propos. L'animateur d'une émission hebdomadaire interroge des touristes français ayant expérimenté une formule de séjour dans un club de vacances à l'étranger ; après avoir recueilli une série de témoignages positifs, il s'adresse à la dernière personne du groupe :

(29) - Et vous? - Moi, je regrette de ne pas m'être plus amusée, en revanche.

La généralisation de l'usage de « $\mathrm{A}$, en revanche $\mathrm{B}$ » est à ce prix : les locuteurs concernés l'emploient même là où il est clair que « $B$ » ne représente pas pour eux un « avantage ».

En outre, il nous semble raisonnable d'avancer que l'attitude des puristes à l'égard de par contre, évoquée au début de cette étude, continue de marquer des générations entières de locuteurs, notamment par le biais de la scolarisation; les professeurs de français qui vont jusqu'à interdire à leurs élèves l'emploi de cette expression sont, aujourd'hui encore, sans doute plus nombreux qu'on ne pense - ce qui expliquerait la fréquence, chez beaucoup de sujets parlants, d'une sorte de réflexe 
d'hypercorrection ${ }^{12}$ les conduisant à considérer en revanche comme une variante « soignée » de par contre, qui relèverait, lui, d'un registre « familier » voire « relâché13 » et serait donc « à éviter ».

Le fonctionnement discursif de en revanche décrit ici apparait, dans cette perspective, comme le résultat naturel de l'attitude - consciente ou non - d'un grand nombre de sujets parlants à l'égard de par contre.

\section{Conclusion}

S'appuyant sur l'observation d'un corpus d'énoncés attestés, notre démarche consiste à proposer la caractérisation la plus «globale» possible des termes étudiés et à circonscrire, pour chacun d'eux, l'invariant commun à l'ensemble de ses emplois. On connaît les nombreuses contributions de la «théorie de l'argumentation dans la langue » à la description des connecteurs; c'est dans cette perspective que nous avons voulu situer notre étude de par contre et de la relation qu'il entretient avec en revanche.

Le principe sous-jacent à cette démarche est que leur emploi reflète fondamentalement une dynamique particulière, en mettant des segments de discours dans un type déterminé de relation, et que cette relation n'est autre chose qu'une construction discursive, qu'elle n'existe qu'en discours et par le discours. Dans cette optique, c'est la représentation d'un élément donné comme la contrepartie d'un autre qui constitue - dans notre approche - le point commun à l'ensemble des emplois de par contre.

Abordée sous cet angle, la relation entre par contre et en revanche apparaît comme un lieu de variation linguistique ; faute d'avoir pu mener une enquête sociolinguistique qui aurait permis de vérifier les hypothèses avancées, nous aurons tenté d'en déterminer les paramètres - en nous fondant, là encore, sur la représentation d'un élément donné non seulement comme contrepartie discursive d'un autre élément, mais aussi comme un « avantage pour le locuteur ».

La démarche présentée ici s'inscrit, en effet, dans une conception du discours en tant que représentation, conception qui revient à considérer que l'emploi d'un connecteur a invariablement pour effet de représenter d'une manière spécifique les éléments qu'il relie, et à chercher à rendre compte de l'interprétation des énoncés en accordant une attention particulière à la dynamique discursive qui se met ainsi en place.

Badreddine HAMMA

UFR LLPhi et UMR 7114 (MoDyCo)

Université de Paris $X$ - Nanterre

200, avenue de la République

92001 NANTERRE

\author{
Pierre Patrick HAILLET \\ UFR LSH et UMR 2450 (Métadif, ILF) \\ Université de Cergy-Pontoise \\ 33, boulevard du Port \\ 95011 CERGY-PONTOISE CEDEX
}

\footnotetext{
12 Cf. Gadet, 1989 ; Arrivé, Gadet et Galmiche, 1986 ; Yaguello, 1988.

13 Poids d'une certaine tradition qui considère l'emploi de par contre comme «une façon de parler boutiquière » (Abel Hermant, cité par Grevisse, 1964, p. 994)...
} 
Par contre : un type particulier de dynamique discursive

\section{BIBLIOGRAPHIE}

ADAM, J.-M. (1984), «Des mots au discours : l'exemple des principaux connecteurs », Pratiques n 43, Metz, CRESEF, p. 107-122.

ADAM, J.-M. (1990), Eléments de linguistique textuelle, Liège, Mardaga.

ANSCOMBRE, J.-C. (1985), «Grammaire traditionnelle et grammaire argumentative de la concession », Revue internationale de philosophie $\mathrm{n}^{\circ} 155$, PUF, p. 333-349.

ANSCOMBRE, J.C. et DUCROT, 0. (1976), « L'argumentation dans la langue », Langages n 42, Paris, Larousse, p. $5-27$.

ANSCOMBRE, J.-C. et DUCROT, 0. (1977), « Deux mais en français? », Lingua n 43, p. 23-40.

ANSCOMBRE, J.-C. et DUCROT, O. (1983), L'argumentation dans la langue, Bruxelles, Mardaga.

ARRIVE, M., GADET, F. et GALMICHE, M. (1986), La grammaire d'aujourd'bui, Paris, Flammarion.

DUCROT, 0. (1972), Dire et ne pas dire, Paris, Hermann.

DUCROT, 0. (1980), «Analyse de textes et linguistique de l'énonciation », in 0. Ducrot et al., Les mots du discours, Paris, Minuit, p. 7-56.

DUCR0T, 0. et al. (1976/1980), « Mais occupe-toi d'Amélie », in 0. Ducrot et al., Les mots du discours, Paris, Minuit, p. 93-130.

FLAUX, N. (2001), "Au contraire (de) et le sens de contre », communication au colloque "CONTRE», Université de Metz, actes à paraître dans Recherches linguistiques.

GADET, F. (1989), Le français ordinaire, Paris, Armand Colin.

GREVISSE, M. (1964), Le bon usage, Gembloux, Duculot.

HAILLET, P. P. (2000), « OR, EN TOUT CAS, DE TOUTE FAÇON : contribution de la "théorie de l'argumentation dans la langue" à la pratique de la traduction ", in D. Delas, Traduire 1, CRTH, Amiens, Encrage Edition, p. 187-209.

HAMMA, B. (2001), La préposition PAR : unicité et diversité, mémoire de DEA, Université de Paris X - Nanterre.

HAMmA, B. (à paraître), « PAR ConTRE, en Revanche et MAIS », Université de Paris X - Nanterre.

LEEMAN-BOUIX, D. (1994), Les fautes de français existent-elles?, Paris, Seuil.

MAINGUENEAU, D. (1987), Nouvelles tendances en analyse du discours, Paris, Hachette.

ROSSARI, C. (2000), Connecteurs et relations de discours : des liens entre cognition et signification, Nancy, Presses Universitaires de Nancy.

RUQUET, M. et QUOY-BAUDIN, J.-L. (1988), Comment dire? Raisonner à la française, Paris, Clé International.

WAGNER, R.L., et PINCHON, J. (1962/1991), Grammaire du français classique et moderne, Paris, Hachette.

WIEDERSPIEL, B. et MASSERON, C. (2001), «Contrastivité adverbiale : au contraire, contrairement à, par contre», communication au colloque "CONTRE», Université de Metz, actes à paraître dans Recherches linguistiques.

YAGUELLO, M. (1988), Catalogue des idées reçues sur la langue, Paris, Seuil. 
